Revue d'histoire de l'enfance « irrégulière »

Le Temps de l'histoire

1 | 1998

La protection de l'enfance : regards

\title{
L'Education surveillée en Algérie de 1945 à 1962
}

\section{(2) OpenEdition \\ 12 Journals}

Édition électronique

URL : http://journals.openedition.org/rhei/20

DOI : $10.4000 /$ rhei.20

ISBN : 978-2-7535-1638-0

ISSN : 1777-540X

Éditeur

Presses universitaires de Rennes

Édition imprimée

Date de publication : 15 novembre 1998

Pagination : 156-165

ISSN : 1287-2431

Référence électronique

Raymond Sultan, « L'Education surveillée en Algérie de 1945 à 1962 », Revue d'histoire de l'enfance « irrégulière » [En ligne], 1 | 1998, mis en ligne le 28 juillet 2010, consulté le 04 décembre 2020. URL :

http://journals.openedition.org/rhei/20 ; DOI : https://doi.org/10.4000/rhei.20

(c) PUR 


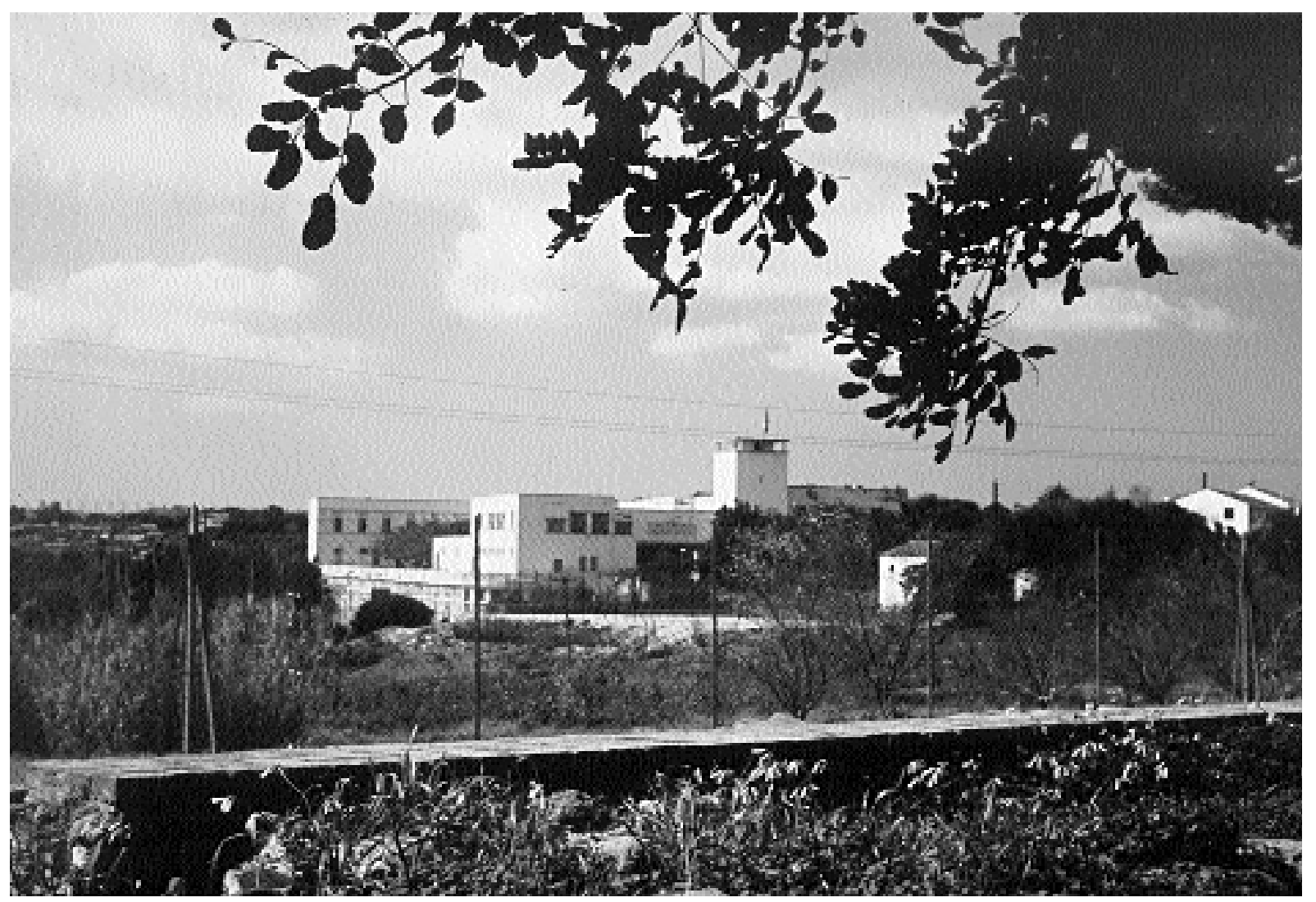

IPES de Birkadem (Algérie)

[Hélène Martignac, 1962] 


\section{L'Éducation surveillée en Algérie de 1945 à 1962}

\section{Raymond Sultan ${ }^{(1)}$}

(1) Éducateur à

l'Éducation surveillée en Algérie, 1951-1962.
Il faut souligner, en préambule, qu'il y a toujours un décalage dans le temps pour l'application en Algérie des textes régissant la rééducation et la protection judiciaire des mineurs en métropole.

C'est ainsi que la loi de 1912 sur les tribunaux pour enfants et adolescents et la liberté surveillée n’a été appliquée en Algérie qu'en 1935. Il a fallu attendre 23 ans pour rendre applicable à l'Algérie une législation pénale qui a substitué, pour les mineurs, des mesures éducatives aux mesures répressives, qui a créé pour eux des juridictions spéciales et institué le régime de liberté surveillée.

Mais comme toujours, le législateur, soucieux de gommer un particularisme suspect, a totalement négligé les contraintes de la réalité culturelle, économique et sociale algérienne et ignoré l'obligation de créer une infrastructure indispensable pour la mise en vigueur du nouveau code de la jeunesse délinquante.

La liberté surveillée, en particulier, n'a pas pu s'organiser de façon efficace et, en 1948, sur les 4.592 mineurs traduits devant les tribunaux des départements d'Algérie, 136 seulement ont été soumis au régime de la liberté surveillée. La proportion, toujours comprise entre 2 et $4 \%$, prouve que le système n'a pu fonctionner pleinement.

Le recrutement des délégués permanents rencontrant des difficultés, c'est l'équipe sociale près le Tribunal d'Alger qui a dû longtemps prendre en charge, avec des délégués bénévoles, les mesures de liberté surveillée. Quant à l'ordonnance du 2 février 1945, charte de la jeunesse délinquante, qui a abandonné en particulier les notions de discernement et de non discernement, pour installer définitivement la prise en 
(2) Ces données judiciaires sont tirées des rapports annuels de la direction de l'Éducation surveillée au Garde des sceaux, de 1954 à 1961. charge des enfants de justice dans le cadre de l'éducation, de la pédagogie, de la psychologie et de la sociologie, elle n'est entrée en vigueur en Algérie qu'en 1951.

Mais là encore, le dilemme, source d'attentisme, a prévalu. L'enfant algérien était-il avant tout un enfant relevant de la psychologie fondamentale de l'enfant et des techniques éducatives métropolitaines? $\mathrm{Ou}$ bien fallait-il s'en tenir à la spécificité des jeunes d'Algérie dans leur contexte national, linguistique, social, économique, moral, religieux? Fallait-il former les éducateurs d'Algérie à l'École nationale de formation des éducateurs à Vaucresson ou créer en Algérie une école d'éducateurs algériens qui construirait une doctrine rééducative propre au pays? Dans ce débat extrêmement délicat, interviennent des notions politiques qui ne sont pas notre propos : assimilation, intégration, autonomie...

Dans le secteur privé, une école d'éducateurs a été créée en Algérie, mais les premiers et derniers éducateurs algériens du secteur public ayant reçu une formation complète l'ont reçue à Vaucresson.

Que représentait l'Éducation surveillée avant l'indépendance de l'Algérie?

\section{DONNÉES JUDICIAIRES ${ }^{(2)}$}

Jusqu'au 1er octobre 1951, date de l'application à l'Algérie de l'ordonnance du 2 février 1945, pour les mineurs jugés, la peine l'emporte sur la mesure éducative. L'année 1951 marque un net fléchissement du pourcentage des mineurs condamnés. Sur une moyenne de 5.000 mineurs jugés par an (avec une pointe importante de près de 8.000 en 1949), le pourcentage de mineurs condamnés passe de 49,40\% en 1949 (près de la moitié) à $44,10 \%$ en 1950 , puis à $30,76 \%$ en 1951 , année où, pour la première fois, le nombre des mesures éducatives prononcées par les tribunaux l'emporte sur celui des condamnations pénales : 2606 mesures éducatives contre 1359 condamnations.

Par la suite, le nombre de mineurs jugés n'a fait que décroître pour passer de 4.417 en 1951 à 2.833 en 1959, alors que la proportion des peines se stabilise autour de $30 \%$, demeurant plus importante qu'en métropole. 
En 1959, pour 2.833 mineurs jugés :

- $14 \%$ ont moins de 13 ans

$-36 \%$ ont de 13 à 16 ans

$-50 \%$ ont de 16 à 18 ans

La proportion des filles est de $5,22 \%$

La même année, les infractions se répartissent ainsi :

- contre les personnes

$25,50 \%$

- contre les biens $36,20 \%$

- contre les mœurs $5,20 \%$

- infractions diverses $33,10 \%$

\section{Dans l'Algérois}

\section{LES ÉTABLISSEMENTS}

\section{Pour les garcons}

L'ancêtre de ces établissements était l'IPES de Birkadem, près d'Alger, un ancien pénitencier militaire désaffecté, qui souffrait, à l'époque de l'après-guerre, de son héritage pénitentiaire et d'un encombrement tel qu'il y avait 400 jeunes pour moins de 200 places.

Par la suite, un effort extrêmement sérieux avait porté essentiellement sur l'aménagement des locaux scolaires, professionnels, de vie de groupe, et cette vie de groupe a été progressivement réorganisée pour atteindre les mêmes performances qu'en métropole. La capacité d'accueil a été ramenée à 160 .

Le Centre d'observation de Birkadem, recevait des mineurs délinquants, vagabonds, moralement abandonnés, ou en "correction paternelle ", de 13 à 18 ans.

Le 27 mars 1947, date mémorable dans les annales de l'Éducation surveillée en Algérie, 100 mineurs âgés de 14 à 18 ans quittent "Barberousse", la grande prison d'Alger, pour le Centre de Birkadem, qui vient d'ouvrir ses portes sur le domaine de l'IPES. La capacité d'accueil de 250 au départ a été ramenée progressivement à 75 , après que ses caractéristiques pénitentiaires aient disparu : grilles d'accès supprimées, cellules individuelles abandonnées, ameublement renouvelé, disparition 
du groupe dit "spécial" où étaient réunis les jeunes poursuivis pour délits politiques et délits de mœurs.

L'Internat approprié de Châteauneuf, à $6 \mathrm{~km}$ d'Alger, a été ouvert le 16 avril 1947. Il reçoit, en principe jusqu'à 14 ans, des enfants de moins de 13 ans, à des fins de formation scolaire et de réadaptation sociale. Il a démarré avec 5 enfants en 1947, puis l'effectif a atteint 79 le 31 décembre 1948, pour se fixer autour d'une centaine, avec un projet de réalisation d'un maximum de 120 places. Mais la prise en charge éducative après l'âge de 14 ans y a toujours posé un problème aigu.

\section{Pour les filles}

Le quartier spécial du groupe pénitentiaire de Maison-Carrée, près d'Alger, qui recevait des filles de 13 à 21 ans, avec un effectif de 60, a été progressivement remplacé par l'IPES de Chéragas, ouverte en 1953, avec un effectif de 48 et une capacité totale d'environ 80 filles de 14 à 18 ans, délinquantes, vagabondes, prostituées, cas sociaux, en général filles difficiles, voire très difficiles.

\section{En Oranie}

Le Centre d'observation d'Oran se situait dans un quartier de la prison construite en 1938. Le nombre de mineurs accueillis oscillait entre 80 et 100 , avec un effectif optimal de 60 .

Mais, en 1960, cet effectif a chuté pour descendre à 28 , alors que le Centre d'observation n'était plus qu'une unité administrative, les garçons ayant été répartis entre l'Internat approprié de Gambetta et l'IPES d'Arzew. À la maison d'arrêt ne restait plus qu'un "quartier spécial" avec 40 condamnés, mineurs de 18 ans et jeunes détenus de moins de 25 ans.

L'Internat approprié d'Oran (ex "Abri Gambetta") a été aménagé avec les moyens du bord (une équipe de mineurs du $\mathrm{CO}$ sous la direction d'un instructeur technique) pour recevoir une quarantaine d'enfants d'âge scolaire.

L'IPES d'Arzew, établissement pavillonnaire moderne, est entré en service en octobre 1955, pour recevoir une centaine de garçons de plus de 13 ans, avec un programme de construction qui aurait dû lui permettre d'atteindre l'effectif de 180 . 


\section{Dans le Constantinois}

L'unique établissement public était le Centre d'observation de Constantine, situé à l'intérieur de la prison, dans des locaux utilisés de façon "ingénieuse", selon Henri Michard. ${ }^{(3)}$ Il recevait, en 1954, 77 garçons. L'avant-projet de construction d'un véritable centre de 100 élèves n’a pu être réalisé.

\section{Au total}

Quand on additionne les effectifs moyens des jeunes pris en charge dans les établissements publics d'éducation surveillée d'Algérie, on arrive à un total de 700 . Face à ce nombre, il faut préciser qu'au moment de l'indépendance de l'Algérie 158 agents de l'Éducation surveillée d'Algérie ont été affectés dans les établissements et services d'éducation surveillée métropolitains.

Ne restaient plus en Algérie, au titre de la coopération, qu'une dizaine d'agents qui, à une seule exception près (et encore s'est-il agi d'un délégué permanent à la liberté surveillée), ont fini par quitter le pays.

Les conditions d'exécution des mesures de liberté surveillée étaient considérablement compromises par ce qu'on appelait pudiquement «les événements ». Pour avoir un ordre d'idée quantitatif, le nombre de mises en liberté surveillée ordonnées en 1960 a été de 291 et, au 31 décembre 1960, 582 mineurs délinquants étaient soumis à cette mesure (542 garçons et 40 filles).

Mais qui était donc le jeune pris en charge par l'Éducation surveillée d'Algérie?

\section{DONNÉES GÉNÉRALES}

L'Algérie était une constellation de groupes ethniques différents. Schématiquement, à l'époque, on disait "les indigènes » et "les européens". Il faudrait plutôt dire "les musulmans » et «les non musulmans".

Ceux-ci, les non musulmans, presque tous des citoyens français, présentaient une homogénéité relative grâce à la langue, le français, mais 
(4) Immigrants venus de Mahon, port des Baléares, au début du siècle. les clivages existaient néanmoins sur le plan culturel, religieux et social entre Français de France, Alsaciens, "Mahonnais", ${ }^{(4)}$ Espagnols, Maltais, Siciliens, Italiens, catholiques, protestants, etc., avec une mention particulière pour les juifs qui, à proprement parler, étaient des indigènes, mais s'étaient totalement assimilés à la France, grâce au décret Crémieux de 1870 qui leur octroyait la nationalité française. Pour les musulmans, il faut distinguer, grosso modo, entre arabophones et berbérophones (Arabes et Kabyles, si l'on préfere), en faisant abstraction de groupes individualisés comme les Mozabites ou certains nomades, et en négligeant les différences de coutumes et de dialectes, ainsi que l'insertion dans l'histoire du pays. Il y avait - il y a encore - deux langues et deux cultures, mais un immense facteur d'unité et d'arabisation, l'islam.

Approximativement, on peut dire qu'il y avait en Algérie un non musulman pour neuf musulmans. Cette proportion était à peu près respectée pour les garçons, chez les mineurs de l'Éducation surveillée. En ce qui concerne les filles, le taux de non musulmanes était inférieur, compte tenu du fait que les petites françaises étaient très souvent prises en charge par les "Bons pasteurs" qui n'acceptaient que très peu de musulmanes.

Il faut noter que cette proportion d'un non musulman pour dix musulmans était inversée dans le personnel éducatif où l'on comptait à peu près un musulman pour dix non-musulmans. L'alternative entre assimilation et spécificité s'est posée. Les lois étaient faites pour fabriquer, théoriquement et à longue échéance, des Français. Mais, s’il est vrai que tous les enfants du monde sont avant tout des enfants, le professeur Jean Sutter écrivait : "L'arabophone doit être rééduqué en arabe par des éducateurs dont l'arabe est la langue maternelle ». C'est un point de vue...

\section{LES JEUNES}

L'éducateur non initié à la psychologie de l'enfant musulman s'étonne parfois des sentiments de respect et d'obéissance manifestés. Il s'agit là d'un transfert de l'attitude qui domine la société musulmane, où l'influence familiale est capitale, avec la mère gardienne des traditions et le père détenteur de l'autorité, entraînant respect, déférence et obéissance. 
Dans la hiérarchie des valeurs familiales, il existe une prédominance, quelquefois formelle, des hommes, impliquant pour les garçons un amour-propre très vif, ainsi qu'une organisation de la maison avec une partie réservée aux femmes. À noter aussi une différence importante entre les jeunes des villes et ceux des campagnes, les campagnards étant plus simples, plus sains, alors que les jeunes des quartiers populeux et des bidonvilles sont livrés à l'école de la rue et des promiscuités. Chez les uns et les autres, cependant, domine naturellement un sentiment de pudeur qu'il faut éviter de contrarier.

Les habitudes culinaires sont également différentes des habitudes européennes : couscous, galette, table basse, plat unique, quelquefois sans fourchette, sans couteau et même sans cuillère... Tous les actes de la vie sont marqués d'un caractère religieux, avec les obligations et les valeurs de l'islam, la prière, le jeûne du Ramadan, l'hospitalité, la charité, le respect du pauvre et du faible. Ces valeurs authentiques sont parfois dénaturées ou exacerbées. Par ailleurs, la "ruralité" d'une grande partie de la population musulmane de l'époque et sa dispersion géographique aboutissaient à une quasi impossibilité d'organiser efficacement une action éducative en milieu ouvert, en dehors des centres urbains.

Pour les petits Européens placés dans les établissements, les choses étaient plus simples; ils rencontraient les mêmes rythmes de vie, les mêmes habitudes. Pour le petit Arabe, celui du bled en particulier, c'était la découverte d'un monde nouveau, voire étranger, un lit au lieu d'une natte, une table, des activités quotidiennes nouvelles, des séances de cinéma régulières, la création d'habitudes, de besoins qui ne pouvaient pas toujours être satisfaits lors du retour au douar d'origine.

Paradoxalement la mesure rééducative pouvait ainsi sécréter une nouvelle forme d'inadaptation sociale.

\section{LES COMPORTEMENTS}

En ce qui concerne les délits, le docteur Maurice Porot ${ }^{(5)}$ écrit : « Comme ailleurs, les garçons volent, les filles se prostituent, les uns et les autres vagabondent. ${ }^{(6)}$ Mais le vagabondage et la prostitution, qui ne sont pas des délits, relèvent de l'assistance éducative. 
(6) "Les enfants délinquants en Algérie”, Rééducation, $\mathrm{n}^{\circ} 3$, janvier 1948, et Sauvegarde de l'Enfance, août-octobre 1950.

(7) Cérémonie religieuse du mariage musulman.
Le vagabondage est très rare à la campagne : la famille rurale respecte la loi coranique, le père imposant sa loi morale et chacun se faisant un devoir de recueillir l'orphelin. Le cas est totalement différent dans les villes; de nombreux enfants abandonnés ou vagabonds couchent dans les bains maures, les couloirs d'immeubles, les chantiers et vivent de la vente de cigarettes, de journaux, de leur activité de cireurs de chaussures ou d'autres petits profits. Ils en arrivent à la mendicité, au vol, à la prostitution.

La prostitution est sensiblement évaluée au même pourcentage qu'en France métropolitaine. Ce sont surtout les filles abandonnées et les femmes répudiées qui s'y livrent. Compte tenu de la coutume du mariage à la "fatah", (7) ces femmes répudiées sont souvent mineures. Il s'agit d'unions imposées à la fillette par les parents, avec un mari qu'elle ne connaît pas et qui peut être un enfant comme elle, un adulte, un vieillard même. Ces unions peuvent aboutir à la répudiation, et la femme répudiée, pour ne pas encombrer le foyer paternel d'une bouche inutile, est réduite à la prostitution et devient quelquefois la proie des proxénètes. Une loi de 1930, fixant à quinze ans l'âge minimum du mariage, n'a pratiquement jamais été appliquée.

\section{Le vol}

\section{LES INFRACTIONS}

Il représentait $45 \%$ des délits.

\section{Les violences envers autrui}

- le meurtre, en France et en Algérie, représentait $1 \%$ des infractions.

- les coups et blessures représentaient $5 \%$ des délits en France et $10 \%$ en Algérie. Il faut souligner que quelquefois meurtres, coups et blessures étaient issus, particulièrement à la campagne, des conflits familiaux et s'apparentaient à une sorte de "vendetta", mêlée à l'honneur et à la vengeance.

\section{LES CORRECTIONS PATERNELLES}

Pour en donner une idée, précisons que le nombre de mineurs faisant l'objet d'une correction paternelle a été, en 1959, de 244 (186 garçons 
et 58 filles). Parmi eux, 136 garçons et 45 filles ont fait l'objet d'une mesure de placement, dont 55 en IPES.

\section{UNE CATÉGORIE PARTICULIÈRE}

La guerre d'Algérie a conduit dans les établissements une nouvelle catégorie de jeunes, les "politiques", dont une vingtaine dans la seule IPES de Birkadem. Bien entendu, il n'y avait pas officiellement de "politiques", puisque tous les mineurs relevaient du droit commun. Mais personne n'était dupe, et la présence des "politiques" a posé quelques problèmes. Pour la plupart issus de la bourgeoisie musulmane, scolarisés et parlant le français, ils étaient en général respectueux des personnels et des règles de discipline de l'établissement. Mais ils jouissaient d'un certain prestige auprès des autres élèves; et l'on a pu détecter un phénomène de "contamination réciproque", certains petits voleurs devenant des "terroristes", comme on disait, et certains jeunes "politiques" glissant vers la délinquance organisée ou individuelle.

\section{EN GUISE DE CONCLUSION}

Dans ce pays pluriel, l'Algérie, où cohabitaient tant de communautés qui souvent s'ignoraient, les choses étaient loin d'être simples.

Malgré les drames quotidiens, la guerre, les tensions explosives, les personnels de l'Éducation surveillée accomplissaient leur mission dans des conditions difficiles, mais ils l'accomplissaient.

La pluralité aurait-elle pu subsister sous une forme ou une autre dans cette terre d'Afrique?

On ne refait pas l'histoire... 